Article

\title{
Exogenous Melatonin and Abscisic Acid Expedite the Flavonoids Biosynthesis in Grape Berry of Vitis vinifera cv. Kyoho
}

\author{
Mingyi Yang ${ }^{1}$, Lei Wang ${ }^{1}$, Tarun Belwal ${ }^{1}$, Xiaocheng Zhang ${ }^{1}$, Hongyan Lu ${ }^{1}$, Cunkun Chen ${ }^{2}$ \\ and $\mathrm{Li} \mathrm{Li} 1,3,4, * \mathbb{D}$
}

1 Key Laboratory for Agro-Products Postharvest Handling of Ministry of Agriculture, Zhejiang Key Laboratory for Agro-Food Processing, College of Biosystems Engineering and Food Science, Zhejiang University, Hangzhou 310058, China; ymy008@zju.edu.cn (M.Y.); wangley@zju.edu.cn (L.W.); tarungbpihed@gmail.com (T.B.); fyzxc@163.com (X.Z.); luhongyan@zju.edu.cn (H.L.)

2 National Engineering Technology Research Center for Preservation of Agricultural Products, Key Laboratory of Postharvest Physiology and Storage of Agricultural Products, Ministry of Agriculture of China, Tianjin 300112, China; cck0318@126.com

3 Ningbo Research Institute, Zhejiang University, Ningbo 315100, China

4 National-Local Joint Engineering Laboratory of Intelligent Food Technology and Equipment, Zhejiang Key Laboratory for Agro-Food Processing, Zhejiang Engineering Laboratory of Food Technology and Equipment, Zhejiang University, Hangzhou 310058, China

* Correspondence: lili1984@zju.edu.cn; Tel./Fax: +86-571-8898-1885

Received: 13 November 2019; Accepted: 11 December 2019; Published: 18 December 2019

\begin{abstract}
Grape polyphenols contributing to more than half of the global polyphenol market were well studied; however, how melatonin (MLT), a potential plant hormone, and abscisic acid (ABA) affects polyphenols profile is still poorly understood. To explore whether these hormones are involved in polyphenolic biosynthesis, grape (Vitis vinifera cv. Kyoho) was exposed to MLT, ABA, and NDGA (nordihydroguaiaretic acid, an ABA biosynthesis inhibitor) treatments, and 16 polyphenols were identified from grape extracts by high performance liquid chromatography quadrupole time of flight mass spectrometry (HPLC-Q-TOF-MS). Both exogenous MLT and ABA significantly enhanced the biosynthesis of each flavonol and flavanol component, especially catechin, which was almost increased double by $200 \mu \mathrm{M}$ of MLT treatment. Furthermore, the expression of genes involved in flavonoid biosynthesis, including 4-coumaroyl-CoA synthase, chalcone synthase, flavonoid 3'-hydroxylase, anthocyanin $3^{\prime}$-methyltransferase, flavonol synthase, flavonoid-3-O-glucosyltransferase, and flavonoid $3^{\prime}, 5^{\prime}$-methyltransferase were highly up-regulated as well but were down-regulated by NDGA. The present study provided new insights for improving flavonoids accumulation in agricultural production and its underlying mechanism.
\end{abstract}

Keywords: grape; melatonin; abscisic acid; flavonoid; Vitis vinifera

\section{Introduction}

Grapevine (Vitis vinifera L.) is a rich source of natural antioxidant compounds, mainly polyphenols, which are composed of flavonols, flavanols, and anthocyanins and contributed to more than half of the global polyphenol market [1]. Grape polyphenols are important secondary metabolites with various health-promoting effects, including anti-inflammatory, anti-cancer, anti-irradiation, anti-bacterial, preventing cardiocerebrovascular diseases, and so on [2]. Besides, Vitis vinifera L cv. Kyoho is one of the most popular cultivars due to its sweetness, juiciness, and large size, and Kyoho cultivar also contribute significantly to the world fresh table grapes [3]. 
Since first discovered in the Japanese morning glory, melatonin (MLT) has been widely studied in plants and plays an important role in stress resistance and antioxidation [4]. Exogenous MLT treatment delayed fruit senescence and improved postharvest commercial value, like inhibiting fruit softening, weight loss, decay rates, and respiration rate of various fruit. Also, promoted endogenous MLT biosynthesis and antioxidant system were observed in pear [5], strawberry [6], peach [7], banana [8], fruit, etc. As a functional component in wine, MLT also had synergistic health effects with polyphenols and increased the vasodilation and antioxidation activities [9]. In addition, $50 \mu \mathrm{M}$ MLT could significantly increase the lycopene level of tomatoes by 5.8 times [10] and maintain the concentrations of total phenolics, flavonoids, and anthocyanins in litchi fruit, contributing to improved antioxidant capacity [11]. In grape berries, it was reported that pre-harvest exogenous MLT treatment significantly increased the polyphenolic content, antioxidant capacity, and related gene expressions, and improved the fruit maturity [12].

Abscisic acid (ABA) is one of the crucial plant hormones, which play vital roles in fruit ripening and development. Studies reported that exogenous ABA promoted fruit coloration, including anthocyanin and flavanol accumulation during fruit ripening in apple [13], citrus [14], grape [15], litchi [16], strawberry [17], and tomato [18]. It was also reported that the transcriptional levels of phenylalanine ammonia-lyase $(P A L)$, cinnamate-4-hydroxylase $(C 4 H)$, chalcone synthase $(C H S)$, chalcone isomerase $(C H I)$, dihydroflavonol 4-reductase (DFR), leucoanthocyanidin dioxygenase ( $L D O X)$ genes involved in polyphenolic biosynthesis were significantly increased by exogenous ABA treatment [16]. Hu et al. observed that $25 \mathrm{mg} \mathrm{L}^{-1}$ exogenous ABA promoted flavonoid biosynthetic gene expression and maintained the color of litchi pericarp [16]. The nordihydroguaiaretic acid (NDGA), an ABA biosynthesis inhibitor, was evident to be involved in the regulation of anthocyanin biosynthesis [19]. Moreover, the effect of ABA on transcript expression of genes involved in anthocyanin and flavonoid biosynthesis has been confirmed in strawberry [20].

However, the effect of postharvest exogenous MLT, ABA, and NDGA on the performance of polyphenolic biosynthesis in grapevine is unclear. The present study was to elucidate the polyphenol profiles and try to develop its relationship with gene expression in Vitis vinifera cv. Kyoho, in order to provide new insights for the improvement of polyphenol accumulation.

\section{Results}

\subsection{Grape Morphology and Berry TSS, TA Concentrations}

In order to investigate the effects of MLT and ABA on grape acceptance, the morphology of grape bunches at harvest and after storage is shown in Figure 1a. The results showed no obvious difference in the treated grape bunches after three day (d) storage at room temperature. Total soluble solid (TSS), which is a measure for sucrose concentration was found to be non-affected with all treatments, except ABA (Figure 1b), but the total acid (TA) concentration was halved to approximately $0.3 \%$ citric acid equivalents after three d storage (Figure 1c). It was interesting to mention that exogenous MLT at a lower concentrations significantly increased the TA concentration by about $0.15 \%(p<0.05)$ compared to CT (Figure 1c). 
(a)

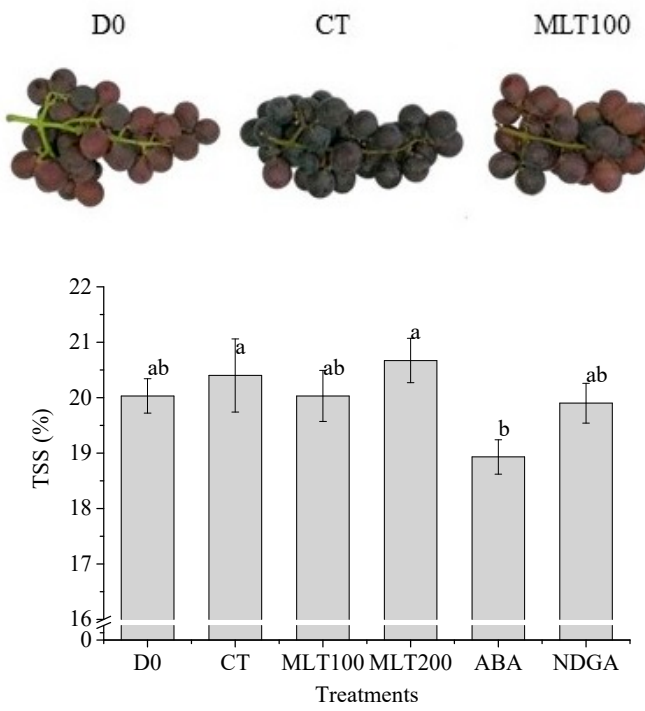

(b)

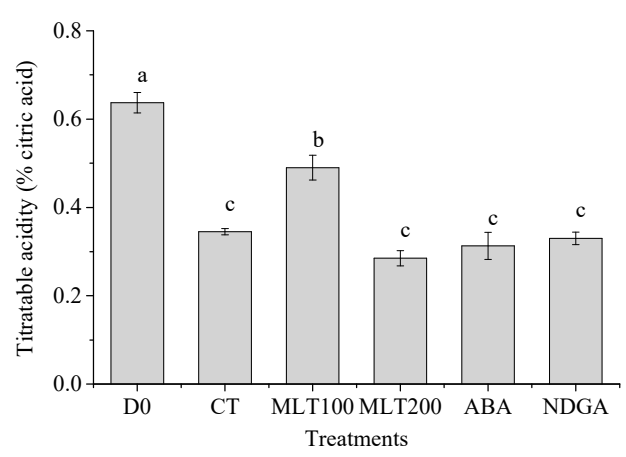

(c)

Figure 1. Grape morphology (a), Total soluble solid (TSS) content (b), and Total acid content (TA) (c) of Vitis vinifera cv. 'Kyoho'. Error bars represent the standard deviations of three replicates. Different letters $(\mathrm{a}-\mathrm{c})$ on the bars represent significant differences between treatments $(p<0.05)$.

\subsection{Polyphenolic Profiles}

To characterize the polyphenol composition and concentration in response to exogenous treatments, the HPLC-Q-TOF-MS method was employed and a total of 18 polyphenol components were detected (Table 1). By comparison to the reported characteristic ion fragments of grape polyphenols, 16 polyphenols were identified, including four phenolic acids, three flavonols, five flavanols, and four anthocyanins. Figure 2 showed the chromatograms of these compounds at UV $280 \mathrm{~nm}, \mathrm{UV} 320 \mathrm{~nm}$, and UV $520 \mathrm{~nm}$. Several mass spectra of typical components of phenolic acid, flavanol, and anthocyanin are shown in Figure 3.

Phenolic acids were found to be the most abundant polyphenolic compound in grapes (Table 1). Out of the four phenolic acids, caftaric acid was found to be the most abundant component, which accounted for more than $85 \%$ of total phenolic acid, followed by fertaric, coutaric, and gallic acid. Consistent with the total phenolic acid content, all identified phenolic acid contents were decreased during storage (Table 1-CT). Among all the treatments, MLT 200 and ABA showed better recovery of all phenolic acids compared to the control (CT). More specifically, MLT200 treatment significantly increased the gallic acid and fetaric acid contents compared to CT $(p<0.05)$. Similarly, NDGA treated grapes showed a significantly higher concentration of gallic acid $(p<0.05)$.

All identified flavonols and flavanols showed significantly lower content after the storage $(p<0.05)$ (Table 1-CT). However, all exogenous treatments showed better recovery of all these components as compared to CT. Among MLT treatments at two different concentrations (100 and $200 \mu \mathrm{M})$, MLT200 showed significantly better recovery of all flavonol and flavanol components $(p<0.05)$ (Table 1$)$. More specifically, the concentrations of catechin and epicatechin and their derivatives were tremendously increased in MLT treatment as compared to CT. A similar trend was recorded in that of laricitrin under ABA treatment. Interestingly, polyphenols, such as gallic acid, laricitrin, Kaempferol 3-O-glucoronide, proanthocyanidin trimer, and (-)-epicatechin-(4beta- $>8)-(-)$-epicatechin/dimeric procyanidin were found to be significantly higher $(p<0.05)$ in the concentration in nordihydroguaiaretic acid (NDGA) treated grapes compared to D0 (Table 1). 
Table 1. Identified polyphenolic compounds and their comparative concentrations $\left(\mathrm{mg} \mathrm{kg}^{-1}\right)$ in the berry of Vitis vinifera cv. 'Kyoho'.

\begin{tabular}{|c|c|c|c|c|c|c|c|c|c|c|c|}
\hline $\begin{array}{l}\text { Peak } \\
\text { No. }\end{array}$ & Compound & $\begin{array}{c}\text { Spectrum } \\
(\mathrm{nm})\end{array}$ & RT (min) & Mw (Da) & Productions & D0 & CT & MLT100 & MLT200 & ABA & NDGA \\
\hline \multicolumn{12}{|c|}{ Phenolic acids } \\
\hline 1 & Gallic acid & 280 & 9.80 & 170 & 125 & $0.85 \pm 0.02^{\mathrm{c}}$ & $0.45 \pm 0.09 \mathrm{e}$ & $0.65 \pm 0.03^{\mathrm{d}}$ & $0.94 \pm 0.03^{b}$ & $0.68 \pm 0.03^{\mathrm{d}}$ & $1.22 \pm 0.00^{\mathrm{a}}$ \\
\hline 2 & Caftaric acid & 320 & 18.15 & 312 & $179,149,135$ & $611.56 \pm 4.70^{\mathrm{a}}$ & $538.68 \pm 17.25^{b}$ & $488.60 \pm 33.41^{b c}$ & $511.59 \pm 8.23^{c}$ & $602.93 \pm 40.67^{\mathrm{a}}$ & $402.73 \pm 2.43^{\mathrm{d}}$ \\
\hline 3 & Coutaric acid & 280 & 23.86 & 296 & 163 & $3.56 \pm 0.04^{\mathrm{a}}$ & $2.83 \pm 0.12^{c}$ & $2.29 \pm 0.07^{\mathrm{d}}$ & $2.96 \pm 0.04^{\mathrm{c}}$ & $3.06 \pm 0.03^{b}$ & $2.08 \pm 0.01^{\mathrm{e}}$ \\
\hline 4 & Fertaric acid & 320 & 27.99 & 326 & 193,134 & $21.28 \pm 0.16^{b}$ & $20.74 \pm 0.43^{c}$ & $17.71 \pm 0.20^{\mathrm{e}}$ & $24.98 \pm 0.15^{\mathrm{a}}$ & $19.67 \pm 0.23^{\mathrm{d}}$ & $20.50 \pm 0.16^{\mathrm{c}}$ \\
\hline \multicolumn{6}{|c|}{ Total phenolic acid content } & $637.25 \pm 4.92^{\mathrm{a}}$ & $562.70 \pm 17.89^{b}$ & $509.25 \pm 33.71^{c}$ & $540.47 \pm 8.45^{b c}$ & $626.34 \pm 40.96^{\mathrm{a}}$ & $426.53 \pm 2.60^{\mathrm{d}}$ \\
\hline \multicolumn{12}{|c|}{ Flavonols } \\
\hline 5 & Laricitrin & 280 & 13.65 & 332 & 169,125 & $3.08 \pm 0.07^{\mathrm{c}}$ & $1.96 \pm 0.32^{\mathrm{e}}$ & $3.46 \pm 0.22^{b}$ & $2.71 \pm 0.01^{\mathrm{d}}$ & $3.58 \pm 0.54 \mathrm{bc}$ & $4.41 \pm 0.16^{\mathrm{a}}$ \\
\hline 6 & Syringetin & 280 & 14.88 & 346 & 183 & $1.50 \pm 0.00^{\mathrm{a}}$ & - & - & $1.16 \pm 0.00^{\mathrm{b}}$ & $0.73 \pm 0.00^{\mathrm{d}}$ & $0.88 \pm 0.00^{c}$ \\
\hline 7 & Kaempferol 3-O-glucoronide & 280 & 34.27 & 462 & $415,311,149$ & $1.94 \pm 0.22^{a}$ & $1.38 \pm 0.04^{\mathrm{b}}$ & $1.34 \pm 0.10^{b}$ & $1.97 \pm 0.12^{\mathrm{a}}$ & $1.50 \pm 0.12^{b}$ & $2.16 \pm 0.10^{\mathrm{a}}$ \\
\hline \multicolumn{6}{|c|}{ Total flavonol content } & $6.52 \pm 0.29^{b}$ & $3.34 \pm 1.73^{\mathrm{e}}$ & $4.80 \pm 0.32{ }^{d}$ & $5.84 \pm 0.13^{c}$ & $5.81 \pm 0.66^{\mathrm{bc}}$ & $7.45 \pm 0.26^{\mathrm{a}}$ \\
\hline \multicolumn{12}{|c|}{ Flavanols } \\
\hline 8 & Proanthocyanidin trimer & 280 & 13.26 & 866 & $\begin{array}{l}866,865,695 \\
575,451,407 \\
287,243,125\end{array}$ & $2.43 \pm 0.05^{b}$ & $1.34 \pm 0.01^{f}$ & $1.38 \pm 0.03^{\mathrm{e}}$ & $2.01 \pm 0.01^{c}$ & $1.45 \pm 0.01^{\mathrm{d}}$ & $2.96 \pm 0.01^{\mathrm{a}}$ \\
\hline 9 & $\begin{array}{l}\text { (-)-epicatechin-(4beta->8)- } \\
\text { (-)-epicatechin/dimeric } \\
\text { procyanidin }\end{array}$ & 280 & 18.42 & 578 & $425,407,289,245$ & $10.94 \pm 0.77^{\mathrm{a}}$ & $6.05 \pm 0.58^{d}$ & $5.42 \pm 0.36^{\mathrm{d}}$ & $8.25 \pm 0.51^{b}$ & $8.32 \pm 0.00^{\mathrm{b}}$ & $7.23 \pm 0.16^{c}$ \\
\hline 10 & $\begin{array}{l}\text { Catechin } \\
\text { (-)-epicatechin-(4beta->8)- }\end{array}$ & 280 & 20.91 & 290 & 245 & $13.57 \pm 0.29^{\mathrm{a}}$ & $4.20 \pm 0.02^{\mathrm{f}}$ & $5.60 \pm 0.30^{\mathrm{e}}$ & $8.24 \pm 0.15^{c}$ & $7.13 \pm 0.28^{d}$ & $8.64 \pm 0.18^{b}$ \\
\hline 11 & $\begin{array}{l}\text { (-)-epicatechin/dimeric } \\
\text { procyanidin }\end{array}$ & 280 & 25.34 & 578 & $425,407,289,125$ & $4.36 \pm 0.09^{b}$ & $2.37 \pm 0.18^{\mathrm{e}}$ & $2.89 \pm 0.29 \mathrm{~d}$ & $4.06 \pm 0.09^{c}$ & $2.78 \pm 0.12^{\mathrm{d}}$ & $5.16 \pm 0.40^{a}$ \\
\hline 12 & Epicatechin & 280 & 29.02 & 290 & $245,203,109$ & $13.36 \pm 0.62^{\mathrm{a}}$ & $6.67 \pm 0.21^{\mathrm{e}}$ & $8.15 \pm 0.48^{\mathrm{d}}$ & $10.24 \pm 0.49^{c}$ & $8.18 \pm 0.26^{\mathrm{d}}$ & $11.62 \pm 0.43^{b}$ \\
\hline \multicolumn{6}{|c|}{ Total flavanol content } & $44.66 \pm 1.82^{\mathrm{a}}$ & $20.63 \pm 1.00^{\mathrm{f}}$ & $23.44 \pm 1.46^{\mathrm{e}}$ & $32.80 \pm 1.25^{\mathrm{c}}$ & $28.36 \pm 0.67^{\mathrm{d}}$ & $35.61 \pm 1.18^{b}$ \\
\hline \multicolumn{12}{|c|}{ Anthocyanins } \\
\hline 13 & Peonidin 3-O-glucoside & 520 & 23.35 & 463 & 301 & $4.27 \pm 0.06^{\mathrm{d}}$ & $5.52 \pm 0.10^{b}$ & $3.19 \pm 0.25^{f}$ & $6.10 \pm 0.07^{\mathrm{a}}$ & $4.57 \pm 0.09^{c}$ & $3.62 \pm 0.03 \mathrm{e}$ \\
\hline 14 & Malvidin 3-O-glucoside & 520 & 25.50 & 493 & 331 & $0.32 \pm 0.18^{c}$ & $0.83 \pm 0.11^{\mathrm{a}}$ & $0.48 \pm 0.05^{b c}$ & $0.57 \pm 0.05^{b}$ & $0.57 \pm 0.04^{b}$ & $0.45 \pm 0.04^{\mathrm{c}}$ \\
\hline 15 & $\begin{array}{l}\text { Malvidin 3-O-(6"-O-coumaroyl)- } \\
\text { glucoside-5-O-glucoside }\end{array}$ & 520 & 57.32 & 801 & $639,493,331$ & $0.99 \pm 0.01^{b}$ & $1.00 \pm 0.04 \mathrm{ab}$ & $0.55 \pm 0.02^{\mathrm{d}}$ & $1.03 \pm 0.02^{a}$ & $0.84 \pm 0.00^{c}$ & $1.03 \pm 0.06^{\mathrm{ab}}$ \\
\hline 16 & $\begin{array}{l}\text { Peonidin 3-O-(6"-O-coumaroyl)- } \\
\text { glucoside-5-O-glucoside }\end{array}$ & 520 & 58.19 & 771 & $609,463,301$ & $2.67 \pm 0.06^{\mathrm{a}}$ & $2.13 \pm 0.00^{c}$ & $1.20 \pm 0.05^{\mathrm{e}}$ & $2.35 \pm 0.11^{b}$ & $2.22 \pm 0.04^{b}$ & $2.01 \pm 0.02^{\mathrm{d}}$ \\
\hline \multicolumn{6}{|c|}{ Total anthocyanin content } & $8.25 \pm 0.31^{c}$ & $9.48 \pm 0.25^{b}$ & $5.42 \pm 0.37^{\mathrm{e}}$ & $10.05 \pm 0.25^{\mathrm{a}}$ & $8.20 \pm 0.17^{c}$ & $7.11 \pm 0.15^{\mathrm{d}}$ \\
\hline \multicolumn{12}{|c|}{ Unidentified } \\
\hline 17 & Unknown & 280 & 7.49 & 370 & 207 & $6.50 \pm 0.01^{\mathrm{a}}$ & $4.49 \pm 0.19^{d}$ & $4.58 \pm 0.09^{\mathrm{d}}$ & $6.19 \pm 0.12^{b}$ & $4.82 \pm 0.08^{\mathrm{c}}$ & $6.44 \pm 0.04^{\mathrm{a}}$ \\
\hline 18 & Unknown & 280 & 12.86 & 452 & 323,89 & $2.52 \pm 0.21^{\mathrm{a}}$ & $1.36 \pm 0.05^{\mathrm{d}}$ & $1.28 \pm 0.09^{d}$ & $2.02 \pm 0.07^{b}$ & $1.79 \pm 0.01^{\mathrm{c}}$ & $1.69 \pm 0.18^{c}$ \\
\hline & Total polyphenol content & & & & & $705.70 \pm 7.56^{\mathrm{a}}$ & $602.00 \pm 21.11^{b}$ & $548.77 \pm 36.04^{\mathrm{c}}$ & $597.37 \pm 10.27^{b}$ & $675.32 \pm 42.55^{\mathrm{a}}$ & $484.83 \pm 4.41^{\mathrm{d}}$ \\
\hline
\end{tabular}

Data are shown in mean \pm standard deviation ( $\mathrm{n}=3$ ). D0, at harvest; CT, control; MLT100, $100 \mu$ M melatonin treatment; MLT200, $200 \mu \mathrm{M}$ melatonin treatment; ABA abscisic acid treatment; NDGA, nordihydroguaiaretic acid treatment. Different letters (a-e in the table) of the same component under different treatments represent statistically significant differences $(p<0.05)$. 

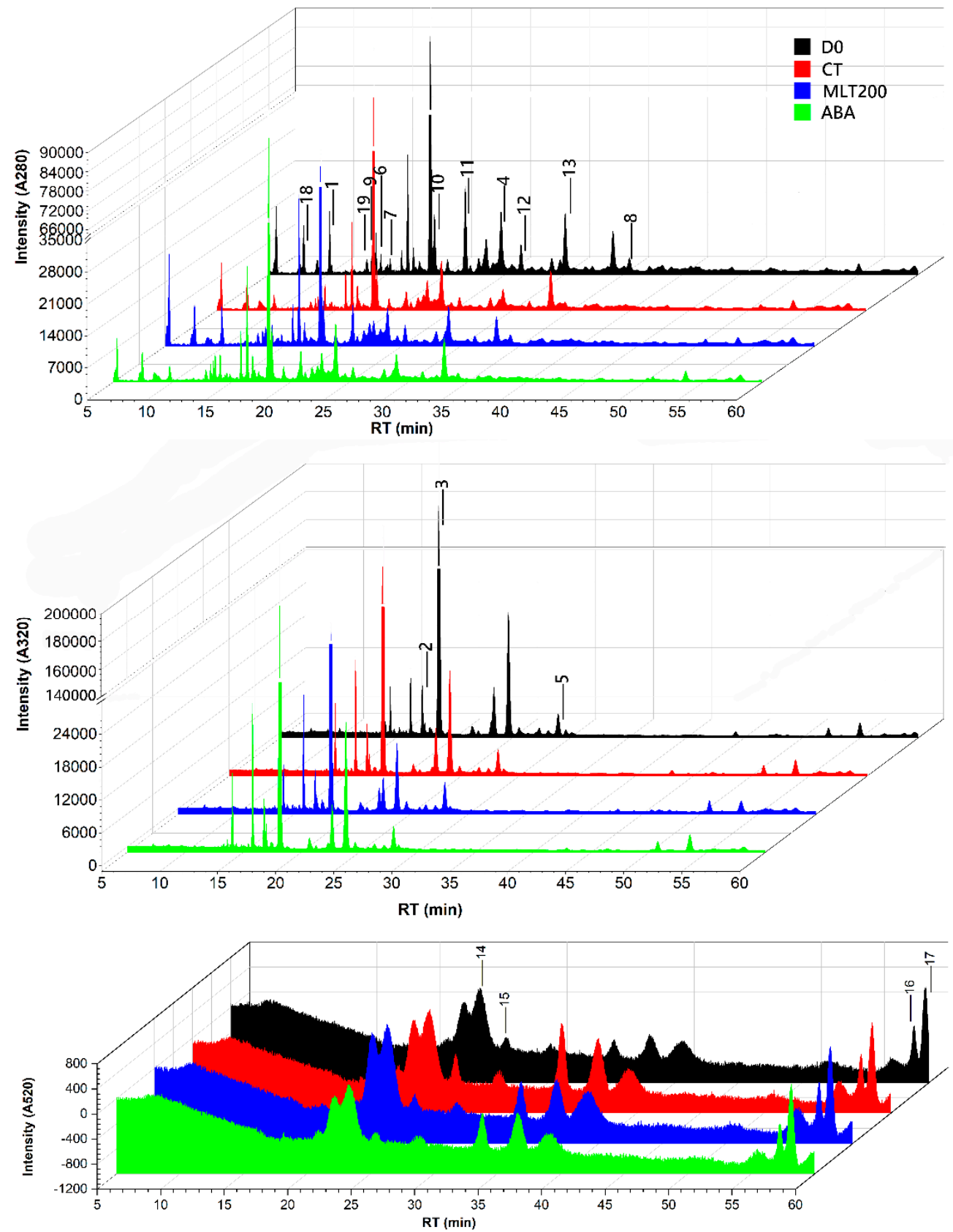

Figure 2. Identification of individual polyphenols in the berry of Vitis vinifera cv. 'Kyoho'. HPLC chromatograms recorded at 280,320, and $520 \mathrm{~nm}$ of the grape extract. The numbered identified peaks are listed in Table 1. To avoid confusion caused by overlapping pictures, only data of D0 (at harvest), CT (control), MLT200 (200 $\mu \mathrm{M}$ melatonin treatment), and ABA (abscisic acid) groups with better effects on polyphenolic biosynthesis promotion were presented in this figure. 

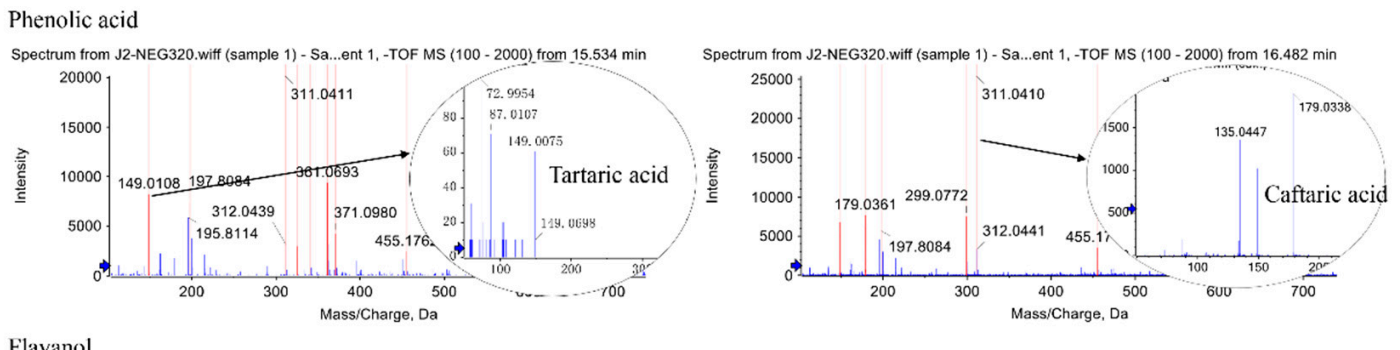

Flavanol
Spectrum from J2-NEG280.wiff (sample 1) - Sa...ent 1, - TOF MS (100 - 2000) from 20.590 min
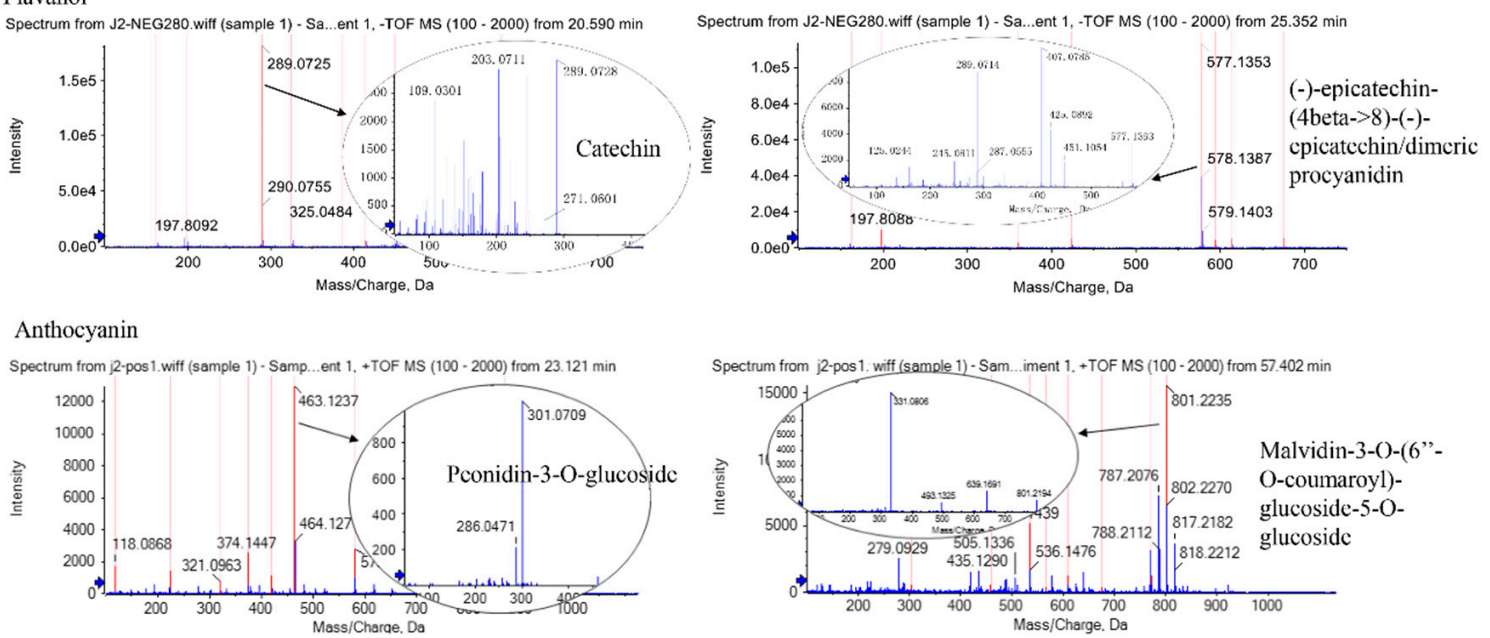

Figure 3. Mass spectra of typical components of phenolic acids, flavanols, and anthocyanins in the berry of Vitis vinifera cv. 'Kyoho'. The circle at the arrowhead is the secondary mass spectrum of the corresponding substance.

Four anthocyanin compounds were determined, primarily consisted of malvidin and peonidin (Table 1). In agreement with the profiles of total anthocyanin content, the lower concentration of exogenous MLT significantly decreased the content of individual anthocyanins $(p<0.05)$. Furthermore, the peonidin-3-O-glucoside compound, which accounted for more than half of the total anthocyanins in the 'Kyoho' berry, was found to significantly increase in MLT200 treatment as compared to CT $(p<0.05)$.

\subsection{Total Phenolic Acid, Flavonol, Flavanol, and Anthocyanin Contents}

Polyphenolic content, reflecting the antioxidant capacity of grape berry, is critical to its quality evaluation, particularly to juices and wines. Our results revealed that total phenolic acid content was declined to $562.70 \mathrm{mg} \mathrm{kg}^{-1}$ in control after three day (d) storage. Almost all exogenous treatments except ABA was found to significantly decrease the total phenolic acid in grapes $(p<0.05)$ (Table 1$)$. Moreover, since total polyphenol content was mainly determined by phenolic acid, its trend was basically consistent with that of phenolic acid (Table 1). Table 1 showed that the total flavanol wshalf-lost after the storage; however, in the exogenous treated grapes, the contents were significantly recovered $(p<0.05)$ and highest were recorded in NDGA $\left(35.61 \pm 1.18 \mathrm{mg} \mathrm{kg}^{-1}\right)$ followed by MLT200 $\left(32.80 \pm 1.25 \mathrm{mg} \mathrm{kg}^{-1}\right)$ treated grapes. The total content of flavonol varied similar to that of flavanol. Anthocyanin content is mainly responsible for the color of the fruit and played a major role in wine formulation. Total anthocyanin content was found to be increased in the control group as compared to D0, which was maintained in ABA treated grapes (Table 1). It was interesting to report that MLT200 showed significantly $(p<0.05)$ higher concentration of total anthocyanin content $\left(10.05 \pm 0.25 \mathrm{mg} \mathrm{kg}^{-1}\right)$ compared to control and all other treatments. 


\subsection{Expression of Genes Involved in Polyphenolic Biosynthesis}

To better understand how MLT and ABA-induced polyphenol anabolism, RT-qPCR was performed to investigate the gene expression profiles of enzymes involved in polyphenolic biosynthetic pathways (Figure 4). The results indicated that the flavonoid biosynthesis in the 'Kyoho' berry was suppressed by the down-regulation of gene expression levels after storage (CT), which was inconsistent with the change in total polyphenol content. Furthermore, almost all gene expression levels were up-regulated by exogenous ABA treatment, except for anthocyanidin 3-O-glucosyltransferase (3GT) and anthocyanidin reductase (ANR); and the upstream flavonoid biosynthetic genes, including 4-coumaroyl-CoA synthase $(4 C L)$, flavonoid $3^{\prime} 5^{\prime}$-hydroxylase $\left(F 3^{\prime} 5^{\prime} H\right), L D O X$, anthocyanin $3^{\prime}$-methyltransferase $(O M T)$, and flavonoid $3^{\prime}, 5^{\prime}$-methyltransferase $(A O M T)$, showed higher transcript levels. On the contrary, most gene expressions in the NDGA group were down-regulated in comparison with ABA. Additionally, most gene expression levels were increased by exogenous MLT, especially OMT, flavanol synthase (FLS), and AOMT genes, which were in agreement with the accumulation of laricitrin and syringetin compounds. And the expression of other genes like $C H I$, flavanone $3 \beta$-hydroxylase (F3H), DFR, LDOX, and ANR were inhibited by both MLT100 and MLT200 treatments.

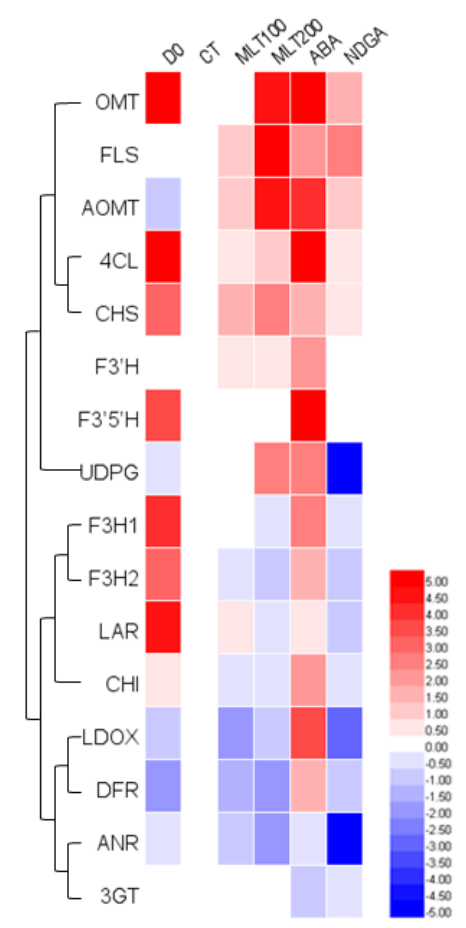

Figure 4. Heatmap of gene expression profiles involved in polyphenolic biosynthesis in the berry of Vitis vinifera cv. 'Kyoho'. The log2 of relative expression levels are shown, measured by RealTime Quantitative Polymerase Chain Reaction (RT-qPCR). CT was set as control.

\section{Discussion}

Grapes are known to be one of the richest sources of various health-promoting compounds, especially polyphenols $[1,21]$. Vitis vinifera cv. 'Kyoho' variety is known for its easy skin peeling effect and considered to possess higher polyphenolic content, thus widely used in wine formulation. In general, our present research results revealed positive changes in polyphenolic content after various postharvested exogenous treatments of grape.

For fresh-eating, it is essential to check the level of TSS and TA contents before harvesting. TSS and TA are important criteria which determine the maturity and taste. All exogenous treatments in the present study showed different levels of TSS and TA contents. Among all exogenous treatments, MLT maintained the TSS level in postharvested grape, while only a lower concentration of MLT showed 
significant increase in TA content as compared to control ( $p<0.05$, Figure 1$)$. This might be due to the fact that melatonin inhibited grape respiration, slowed down citric acid circulation, and reduced the degradation of soluble acid, which was confirmed in sweet cherry [22]. The color indicates the ripening stage and berry development. A noticeable change in the berry color was recorded in the control group, while in other exogenous treated groups, it was less predominant. It was previously reported that MLT spraying reduced the under-ripeness and over-ripeness during grape pre-veraison, promoting the berry ripening synchronicity and wines made with it were more fruity, spicy, and sweet [23].

Hilbert et al. [24] identified and detected structures of 18 flavanols from six different Vitis varieties using liquid phase mass spectrometry (LC-MS) and liquid chromatography-nuclear magnetic resonance spectroscopy (LC-NMR), respectively. In order to elucidate the effect of these exogenous treatments on polyphenolic profiles of in Vitis vinifera cv. 'Kyoho' berry during storage, we detected a total of 18 polyphenolic compounds and identified 16 by HPLC-Q-TOF-MS, and determined their relative quantitatively concentrations by HPLC, and investigated the gene expressions involved in the polyphenolic biosynthetic pathway. Moreover, the exogenous melatonin was widely applied in fruit to improve its quality. For instance, grape treated with MLT at pre-veraison stage possessed higher levels of catechins, epicatechins, and peonidin derivatives after maturation [25]. This is consistent with our finding that MLT significantly promoted catechin content $(p<0.05)$. Moreover, the fruit quality and yield of tomato were improved by exogenous MLT, and the contents of phenolics and flavonoids in green-mature tomato were increased by $14.29 \%$ and $30.77 \%$, respectively, which were almost twice than that in the red-mature tomato [26]. These results suggested that the polyphenolic biosynthesis was slowed down during fruit senescence, in accordance with the decrease of total polyphenol content in CT after storage (Table 1). In the present study, the polyphenolic contents (phenolic acids, flavonols, flavanols, and anthocyanins) showed differences among different treatments and within polyphenolic contents (Table 1). For example, MLT 200 was found to maintain the total phenolic acid content, while ABA treatment showed an increase in its concentration. However, in the case of total flavonol and flavanol content, NDGA was found to be the best followed by MLT200 and ABA. Anthocyanin, which is mainly responsible for the fruit color, was found to be up-regulated during MLT200 treatment. MLT at lower concentrations was not found much effective in regulating polyphenolic content as compared to a higher concentration. Overall, the total polyphenol content was found to be declined after postharvested storage condition (CT), possibly due to the oxidation process, but maintained and up-regulated by exogenous treatment, especially MLT200 and ABA, respectively (Table 1). This might be attributed to the improved antioxidant capacity in 'Kyoho' berry by MLT or ABA, which was further evident in litchi [11] and strawberry [6].

Additionally, both the polyphenol accumulation and $P A L$ expression were observed to be promoted by pre-harvest MLT [12]. In agreement with former studies, our RT-qPCR results showed that 4CL, CHS, F3' $H, O M T, F L S, U D P G$ and AOMT genes involved in flavonoid biosynthesis were more expressed in different degrees under MLT treatment (Figure 5). In tomato, ABA significantly promoted carotenoid and flavonoid biosynthesis via up-regulating the expression of related genes by 2.08 to 35 times, initiating polyphenol accumulation 2-4 d earlier than control [27]. It was reported that exogenous treatment of ABA in strawberry accelerated flavonoid and anthocyanin biosynthesis, as well as the up-regulated $C H S, C H I, F 3 H$, flavonoid $3^{\prime}$-hydroxylase $\left(F 3^{\prime} H\right)$, and DFR expression [20]. And Olivares et al. [28] found that exogenous ABA significantly promoted grape coloration and advanced the harvest time by $37 \mathrm{~d}$. In the present study, total polyphenol content, especially phenolic acid, flavonols, and flavanols were also increased by ABA treatment (Table 1). Particularly, it was revealed that all individual flavonoid compounds except anthocyanins exhibited higher content in ABA and NDGA groups than control (Table 1). Accordingly, 4CL, CHS, OMT, FLS, and AOMT genes involved in polyphenolic biosynthesis showed higher expression in ABA and NDGA groups than control (Figure 5). Expression levels of genes such as $C H I, F 3 H, F 3^{\prime} H, F 3^{\prime} 5^{\prime} H, D F R, L D O X$, leucoanthocyanidin reductase $(L A R)$, and flavonoid-3-O-glucosyltransferase (UDPG) were significantly up-regulated in response to exogenous ABA; however significantly and adversely lower in response to NDGA $(p<0.05)($ Figure 5). 
A similar outcome was also found in MLT groups, in which not all genes involved in flavonoid biosynthesis had higher expression levels (Figure 4). This paradoxical phenomenon might be due to the profiles of accumulated flavonoids, which were determined from not only the involvement of key genes in the biosynthesis pathway, such as $4 C L, C H S, O M T, F L S$ and AOMT; but also, from the blocking of the ABA biosynthesis pathway by NDGA. It was reported that the polyphenolic biosynthesis and the internal browning in postharvest pineapple were inhibited by the application of $380 \mu \mathrm{M} A B A$, due to the improved activity of antioxidant enzymes [29].

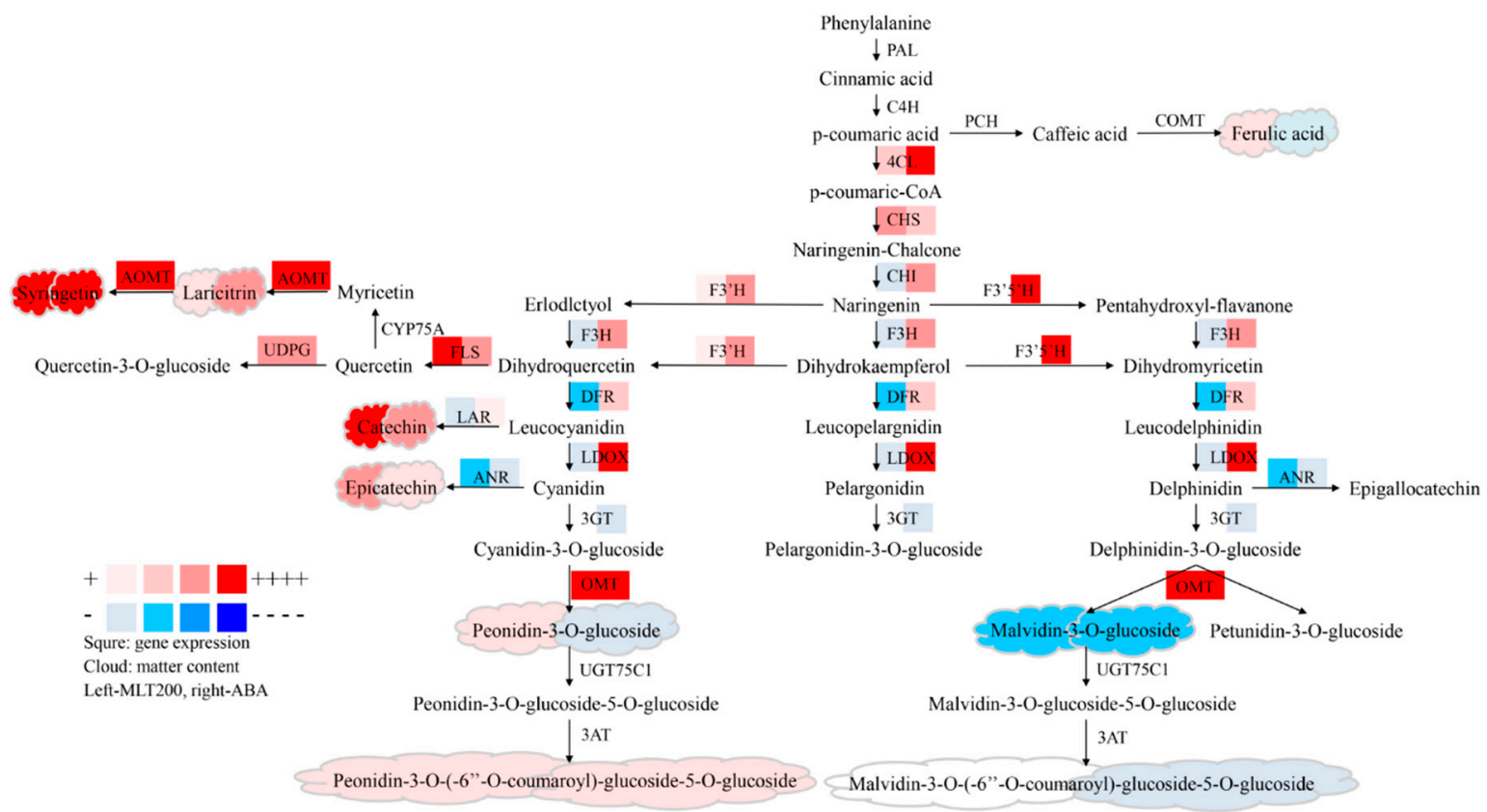

Figure 5. Polyhenolic biosynthesis pathway in berry of Vitis vinifera cv. 'Kyoho'. Red represents up-regulation, while blue represents down-regulation, and white indicates no effect. The deeper the color, the larger the change. And the left reveals MLT200 effect, whereas the right indicates ABA effect. The square and cloud shapes indicate the change in gene expression and component content, respectively.

It was notable to mention that both MLT and ABA promoted flavonoid biosynthesis; therefore, is there any possible interaction between the influences resulted from exogenous MLT and ABA? Changes of polyphenol contents and gene expressions of vital enzymes involved in polyphenol biosynthesis under ABA and MLT treatments were presented in Figure 5. ABA has been widely recognized as a promotor to polyphenolic biosynthesis, and the increase in the expression of ABA receptor VIPYL1 led to the accumulation of anthocyanin and a series of ABA-responsive gene transcripts in grape berries [30]. The expression of key enzymes in the anthocyanin biosynthesis pathway, including $P A L$, C4H, CHS, CHI, F3H, F3' H, DFR, and LDOX, was up-regulated by exogenous ABA in litchi [16] and strawberry [20]. Moreover, the transcription factor MYB could be activated by ABA, then bound to bHLH and WD40 to form a protein complex, which increased anthocyanin biosynthesis [31]. In addition, FLS and UDPG in the flavonoid biosynthesis pathway were higher expressed by ABA as well [18]. Our results from the present study further confirmed the positive role of ABA in fruit flavonoid biosynthesis (Figure 5). Additionally, the first discovery of MLT receptor in Arabidopsis thaliana suggested that MLT was involved in the regulation of physiological attributes, including fruit development and ripening through receptor-mediated signaling cascades [32]. Xu et al. also published that MLT might promote grape ripening by increasing levels of ABA and ethylene [33]. However, very few studies were carried out on the MLT effect on fruit flavonoid biosynthesis so far [12,34]. Our study effectively contributes to narrow down this gap, particularly on grape berries and found that MLT significantly improved flavonoid biosynthesis $(p<0.05)$ via increasing gene expressions of $4 C L$, 
CHS, FLS, AOMT, and UDPG (Figure 5). Enhanced flavonoid contents contribute to higher antioxidant activities, thus contributed to the senescence inhibition, quality maintenance of postharvest fruit. Therefore, we postulated a molecular model on the roles of ABA and MLT in polyphenolic biosynthesis in Vitis vinifera cv. 'Kyoho' berry (Figure 6). Briefly, exogenous ABA promoted the transcription of key enzymes in the main route of polyphenolic biosynthesis pathways, which was then diverged into two roads, leading to higher levels of flavonoids, including anthocyanins. And MLT enhanced flavonoid biosynthesis as well, mainly in a later stage of the pathway. ABA also up-regulated the expression of the MYBA1 (v-myb avian myeloblastosis viral oncogene homolog A1) transcription factor, which further forms a protein complex with transcription factors bHLH and WDR1 to facilitate anthocyanin biosynthesis in grapevine [35]. Increased flavonoid contents contributed to enhanced antioxidant activities and inhibited grape senescence [11]. Our findings provided new insights and made a deeper understanding of flavonoid biosynthesis and gene expression in Vitis vinifera cv. 'Kyoho' berry under exogenous ABA and MLT. Moreover, since ABA and MLT showed similar effects on promoting the flavonoids biosynthesis, their potential interaction will be further explored in our follow-up study.

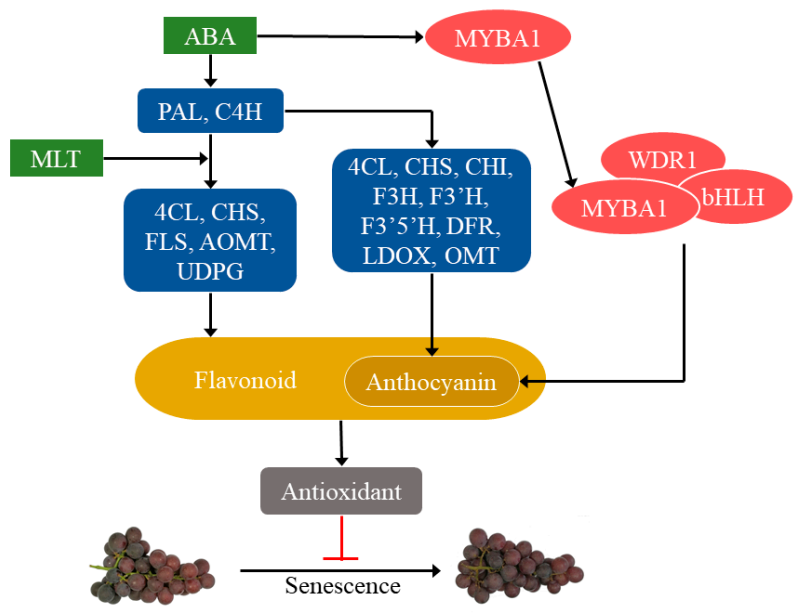

Figure 6. Possible model depicting the mechanism of abscisic acid (ABA) and melatonin (MLT) promoted flavonoid biosynthesis in the berry of Vitis vinifera cv. 'Kyoho' based on previous and present studies.

\section{Materials and Methods}

\subsection{Plant Materials and Experimental Design}

Three independent biological replications of grape (Vitis vinifera cv. 'Kyoho') in August with homogenous size were hand-harvested at the same maturity from an organic vineyard in Jinhua city, Zhejiang province, China. For each biological replication, grape bunches were collected in thermocol boxes and delivered to the laboratory in $1.5 \mathrm{~h}$. After acclimatizing at $25{ }^{\circ} \mathrm{C}$ for $2 \mathrm{~h}$ in the laboratory, the grape bunches were randomly and evenly divided into five groups (three bunches in each group) (Figure 7). Each group was immersed for $20 \mathrm{~min}$ in different exogenous chemicals at varied concentrations, i.e., CT (distilled water, control), MLT100 (100 $\mu \mathrm{M}$ melatonin, Macklin Biochemical Company, Ltd., Shanghai, China), MLT200 (200 $\mu$ M melatonin), ABA (1 mM abscisic acid, Macklin Biochemical Company, Ltd., Shanghai, China) and NDGA (0.5 mM nordihydroguaiaretic acid, Tokyo Chemical Industry, Ltd., Shanghai, China) (Figure 7). Afterward, the grapes were air-dried and stored at room conditions (i.e., $22 \pm 3{ }^{\circ} \mathrm{C}$ temperature and $60 \pm 5 \%$ relative humidity) for three $\mathrm{d}$. Berries were randomly removed for determination of total soluble solids (TSS) and titratable acidity (TA), and other berries, including the skin, the flesh, and seeds were ground, mixed, and frozen in liquid nitrogen and stored at $-80{ }^{\circ} \mathrm{C}$ for further analysis (Figure 7 ). 


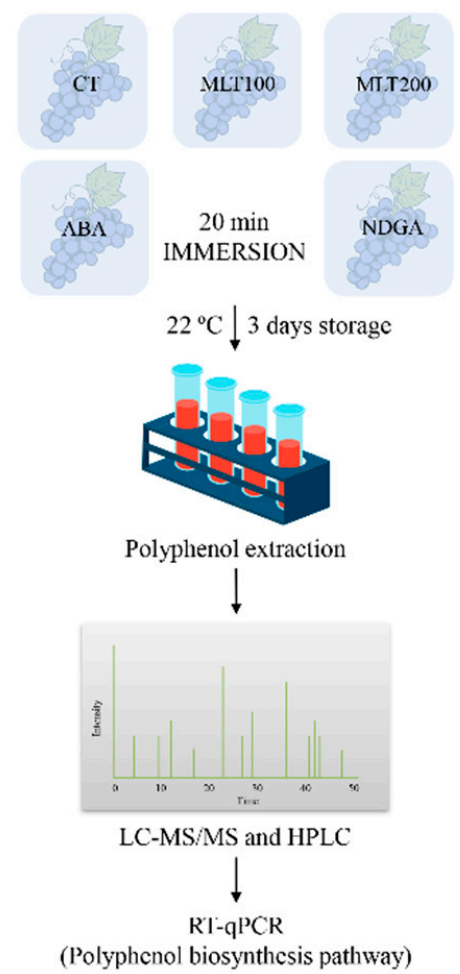

Figure 7. Systematic flow diagram of the experiments.

\subsection{Total Soluble Solids (TSS) and Titratable Acidity (TA) Determination}

TSS and TA of the fruit juice were measured by a Brix-Acidity Meter (Model PAL-BX/ACID F5, Atago, Japan). Three independent replicates were carried out.

\subsection{Polyphenols Extraction and Separation}

Polyphenols in berry (approximately $2.0 \mathrm{~g}$ ) were extracted with $1.0 \mathrm{~mL}$ of $1 \% \mathrm{HCl}$-methanol, at dark $4{ }^{\circ} \mathrm{C}$ for $12 \mathrm{~h}$. The resulting supernatant obtained after centrifugation at $10,000 \times g$ for 15 min was filtered through the $0.22 \mu \mathrm{m}$ microporous membrane. Polyphenolic compounds were separated by injection into the HPLC system (LC-20AD, SHIMADZU incorporation, Japan) with an AQ-C18 column (water-based, $5 \mu \mathrm{m}, 4.6 \times 250 \mathrm{~mm}$, Welch Materials incorporation, Shanghai, China). The elution parameters were as follows: Injection volume, $10 \mu \mathrm{L}$; column temperature, $40{ }^{\circ} \mathrm{C}$ : Solvent $\mathrm{A}, 1 \%$ phosphoric acid; solvent $\mathrm{B}$, acetonitrile; solvent flow rate, $1.0 \mathrm{~mL} \mathrm{~min}^{-1}$; and the gradient: $0-10 \mathrm{~min}$, 2\% B; 10-55 min, 10\% B; 55-65 min, 18\% B; 65-68 min, 50\% B; 68-69 min, 80\% B; 69-79 min, $2 \%$ B. The signal was monitored at 280,320 and $520 \mathrm{~nm}$.

\subsection{Identification and Quantification of Polyphenols by HPLC and HPLC-Q-TOF-MS}

Polyphenols including phenolic acids, flavonoids (including flavones, flavonols, flavanols, flavanones, anthocyanins and so on) were identified using HPLC (as mentioned before) coupled to quadrupole time-of-flight mass spectrometry (Q-TOF-MS) (TripleTOFTM 5600+, SCIEX incorporation, United States) with an electrospray ionization source (ESI) system. Data were generated in negative ion mode with a scan range of $100-1500 \mathrm{~m} / \mathrm{z}$ and the source voltage at $-4.5 \mathrm{kV}$, the source temperature at $550{ }^{\circ} \mathrm{C}$. The pressure of Gas 1 (Air) and Gas 2 (Air) was 50 psi, whereas that of curtain gas $\left(\mathrm{N}_{2}\right)$ was 35 psi. The molecular formula proposed by PeakView software version 1.2 for different signals were compared with previously reported polyphenolic compounds, especially in Vitis vinifera.

The polyphenolic compounds were quantified by comparison of peak areas with standard calibration curves of the gallic acid (Macklin Biochemical Company, Ltd., Shanghai, China) and epicatechin (Aladdin, Shanghai, China) at 280 and $320 \mathrm{~nm}$, and the cyanidin-3-O-glucoside 
(Macklin Biochemical Company, Ltd., Shanghai, China) at $520 \mathrm{~nm}$. The total concentrations of phenolic acid, flavonol, flavanol, and anthocyanin were obtained by the addition of each identified component concentrations.

\subsection{Real-Time Quantitative PCR (RT-qPCR)}

Genes involved in the polyphenolic biosynthesis pathway were selected according to Kyoto Encyclopedia of Genes and Genomes (KEGG) pathway database (https://www.genome.jp/kegg/ pathway.html). The primers of these genes used in this study were listed in Appendix A Table A1. Total RNAs were extracted from powdered grape samples using cetyl trimethyl ammonium bromide (CTAB) protocol described by Gambino et al. [36] and quantified with microplate reader (TECAN, Spark ${ }^{\circledR}$, Männedorf, Switzerland), followed by reverse-transcription to cDNA with a PrimeScript ${ }^{\mathrm{TM}}$ RT Reagent Kit (code DRR047 A, TaKaRa, Japan). RT-qPCR was conducted on TB Green ${ }^{\mathrm{TM}}$ Premix Ex TaqTM (RR420A, TaKaRa, Japan) according to the instructions mentioned in the product. The glyceraldehyde-3-phosphate dehydrogenase (GAPDH) gene was used as a calibrator to calculate the relative transcriptional levels of genes by the $2^{-\Delta \Delta C T}$ method. Three independent replicates were carried out. And the results were presented as a heat map using the Heml 1.0 software [37].

\subsection{Statistical Analysis}

Experiments were completely randomly designed. All data analysis was performed using SPSS V20.0 (IBM Corp, Armonk, NY, USA) software and presented as mean \pm standard deviation with analysis of variance (ANOVA) and Tukey test at a significant level of $p<0.05$.

Author Contributions: Conceptualization, M.Y., L.W. and L.L.; methodology, M.Y. and L.L.; formal analysis, M.Y., X.Z.; investigation, M.Y., L.W. and H.L.; resources, C.C. and L.L.; writing-Original draft preparation, M.Y.; writing-Review and editing, T.B. and L.L.; visualization, M.Y. and L.L.; supervision, L.L.; project administration, L.L.; funding acquisition, L.L. All authors have read and agreed to the published version of the manuscript.

Funding: This research was funded by the National Key Research and Development Program of China (No. 2017YFD0401304) and National Natural Science Foundation of China (No. 31571895, 31772366).

Conflicts of Interest: The authors declare no conflict of interest.

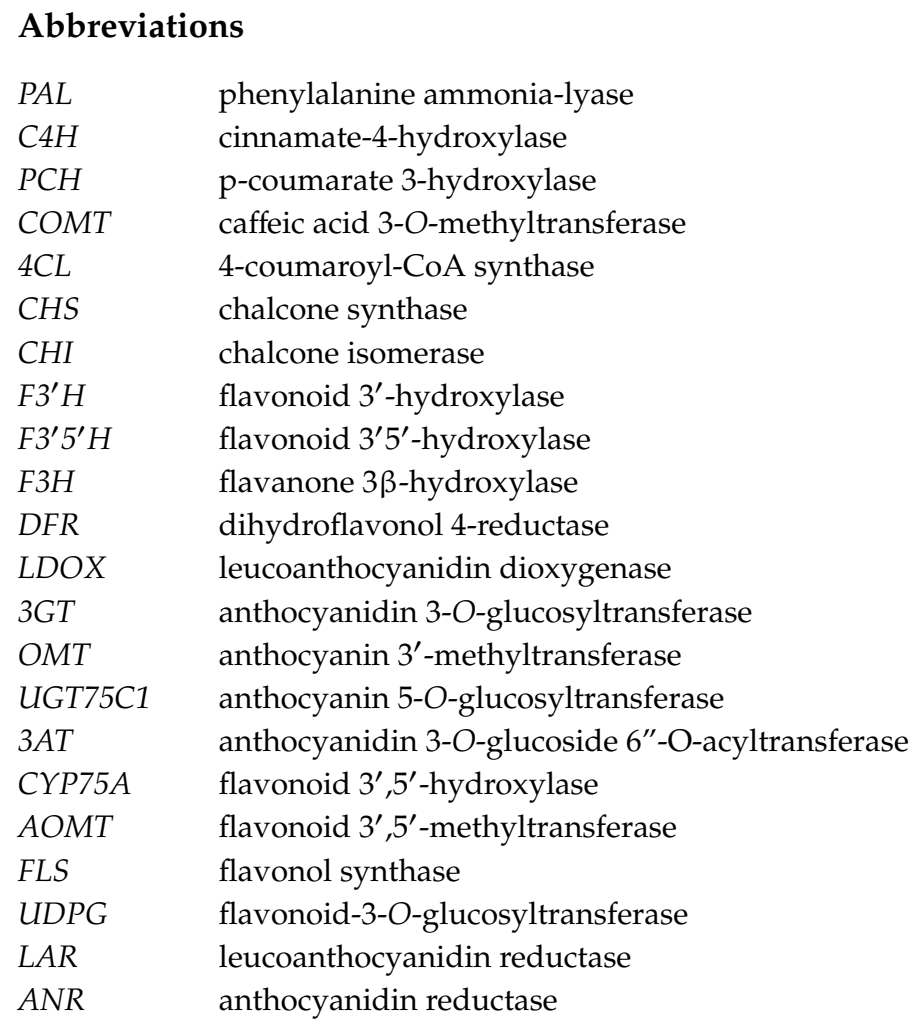




\section{Appendix A}

Table A1. Primers used in RT qPCR.

\begin{tabular}{cll}
\hline Genes & \multicolumn{1}{c}{ Forward Primers $\left(\mathbf{5}^{\prime} \mathbf{-} \mathbf{3}^{\prime} \mathbf{)}\right.$} & \multicolumn{1}{c}{ Reverse Primers $\mathbf{( 5}^{\prime} \mathbf{- 3}^{\prime} \mathbf{)}$} \\
\hline $4 C L$ & ACCACCTCCCTCTCCACAC & GCTCCGAGAAAGGAGAACG \\
$C H S$ & ACCCACCTGGATTCTCTCG & GAAGGCTTCCACCAAGCTC \\
$C H I$ & TTGTGTTGGTTCCTCTTGTTC & GCAGACGAATCTCATTCAGTATAG \\
$F 3 H 1$ & CCAATCATAGCAGACTGTCC & TCAGAGGATACACGGTTGCC \\
$F 3 H 2$ & CTGTGGTGAACTCCGACTGC & CAAATGTTATGGGCTCCTCC \\
$F 3^{\prime} H$ & GAGATCAACGGCTACCACATC & CCTGAATTCTAGTGGCTTCTCC \\
$F 3^{\prime} 5^{\prime} H$ & GCTGGCACTAGAATGGGAATAG & CTCAACTCCATCCGGCATTT \\
$D F R$ & TTGTAATGGTCAATGTGCC & CATGCAGAGACCACCTTG \\
$L D O X$ & ACCTTCATCCTCCACAACAT & AGTAGAGCCTCCTGGGTCTT \\
$3 G T$ & TCATGAAGACCAGACCCTTA & TGTTGCTACTACGGGGTCTA \\
$O M T$ & AGATAAAGAAGCGTACCAAA & GCTCATGGTAGTTGAGGTAG \\
$L A R$ & CGGTTGGTAGCATTAAGAGGTTC & GGAGTTGCAGCAGATGTAGGTGT \\
$A N R$ & TTGATGGGACAGGTCTGGTTG & AGTGTCTTGGAGGCAGGATAGC \\
$F L S$ & CCAGCCAATCCTTCAAACTT & TTTGCCATTTGCATTGTGTA \\
$U D P G$ & TGCTACCTAAGGCGACTG & GCTTGGATTTGAGATCATTGG \\
$A O M T$ & AGGTGTGGAGCATAAGATCA & TTATCGTAAGCGATTATGCC \\
\hline
\end{tabular}

1. Polyphenols Market Projected to Reach $\$ 1.33$ Billion by 2024 . Available online: http: //www.abnewswire.com/pressreleases/polyphenols-market-projected-to-reach-133-billion-by-2024grand-view-research-inc_75694.html (accessed on 11 November 2019).

2. Shahidi, F.; Yeo, J. Bioactivities of phenolics by focusing on suppression of chronic diseases: A review. Int. J. Mol. Sci. 2018, 19, 1573. [CrossRef] [PubMed]

3. Distribution of the World's Grapevine Varieties. Available online: https://www.amorimcorkitalia.com/media/ en-distribution-of-the-worlds-3f7.pdf (accessed on 11 November 2019).

4. Vantassel, D.; Roberts, N.; Oenill, S. Melatonin from higher-plants-isolation and identification of N-acetyl 5-methoxytryptamine. Plant Physiol. 1995, 108, 101.

5. Zhai, R.; Liu, J.; Liu, F.; Zhao, Y.; Liu, L.; Fang, C.; Wang, H.; Li, X.; Wang, Z.; Ma, F.; et al. Melatonin limited ethylene production, softening and reduced physiology disorder in pear (Pyrus communis L.) fruit during senescence. Postharvest Biol. Technol. 2018, 139, 38-46. [CrossRef]

6. Liu, C.; Zheng, H.; Sheng, K.; Liu, W.; Zheng, L. Effects of melatonin treatment on the postharvest quality of strawberry fruit. Postharvest Biol. Technol. 2018, 139, 47-55. [CrossRef]

7. Gao, H.; Zhang, Z.K.; Chai, H.K.; Cheng, N.; Yang, Y.; Wang, D.N.; Yang, T.; Cao, W. Melatonin treatment delays postharvest senescence and regulates reactive oxygen species metabolism in peach fruit. Postharvest Biol. Technol. 2016, 118, 103-110. [CrossRef]

8. Hu, W.; Yang, H.; Tie, W.; Yan, Y.; Ding, Z.; Liu, Y.; Wu, C.; Wang, J.; Reiter, R.J.; Tan, D.X.; et al. Natural variation in banana varieties highlights the role of melatonin in postharvest ripening and quality. J. Agric. Food Chem. 2017, 65, 9987-9994. [CrossRef]

9. Iriti, M.; Varoni, E.M. The good health of Bacchus: Melatonin in grapes, the unveiled myth. LWT-Food Sci. Technol. 2016, 65, 758-761. [CrossRef]

10. Sun, Q.; Zhang, N.; Wang, J.; Zhang, H.; Li, D.; Shi, J.; Li, R.; Weeda, S.; Zhao, B.; Ren, S.; et al. Melatonin promotes ripening and improves quality of tomato fruit during postharvest life. J. Exp. Bot. 2015, 66, 657-668. [CrossRef]

11. Zhang, Y.; Huber, D.J.; Hu, M.; Jiang, G.; Gao, Z.; Xu, X.; Jiang, Y.; Zhang, Z. Delay of postharvest browning in litchi fruit by melatonin via the enhancing of antioxidative processes and oxidation repair. J. Agric. Food Chem. 2018, 66, 7475-7484. [CrossRef]

12. Xu, L.; Yue, Q.; Bian, F.; Sun, H.; Zhai, H.; Yao, Y. Melatonin enhances phenolics accumulation partially via ethylene signaling and resulted in high antioxidant capacity in grape berries. Front. Plant Sci. 2017, 8, 1426. [CrossRef] 
13. An, J.P.; Yao, J.F.; Xu, R.R.; You, C.X.; Wang, X.F.; Hao, Y.J. Apple bZIP transcription factor MdbZIP44 regulates abscisic acid-promoted anthocyanin accumulation. Plant Cell Environ. 2018, 41, 2678-2692. [CrossRef] [PubMed]

14. Wang, X.; Yin, W.; Wu, J.; Chai, L.; Yi, H. Effects of exogenous abscisic acid on the expression of citrus fruit ripening-related genes and fruit ripening. Sci. Hortic. 2016, 201, 175-183. [CrossRef]

15. Villalobos-Gonzalez, L.; Pena-Neira, A.; Ibanez, F.; Pastenes, C. Long-term effects of abscisic acid (ABA) on the grape berry phenylpropanoid pathway: Gene expression and metabolite content. Plant Physiol. Biochem. 2016, 105, 213-223. [CrossRef] [PubMed]

16. Hu, B.; Li, J.; Wang, D.; Wang, H.; Qin, Y.; Hu, G.; Zhao, J. Transcriptome profiling of Litchi chinensis pericarp in response to exogenous cytokinins and abscisic acid. Plant Growth Regul. 2017, 84, 437-450. [CrossRef]

17. Li, D.; Li, L.; Luo, Z.; Mou, W.; Mao, L.; Ying, T. Comparative transcriptome analysis reveals the influence of abscisic acid on the metabolism of pigments, ascorbic acid and folic acid during strawberry fruit ripening. PLoS ONE 2015, 10, e0130037. [CrossRef] [PubMed]

18. Mou, W.; Li, D.; Luo, Z.; Mao, L.; Ying, T. Transcriptomic analysis reveals possible influences of ABA on secondary metabolism of pigments, flavonoids and antioxidants in tomato fruit during ripening. PLoS ONE 2015, 10, e0129598. [CrossRef]

19. Shen, X.; Zhao, K.; Liu, L.; Zhang, K.; Yuan, H.; Liao, X.; Wang, Q.; Guo, X.; Li, F.; Li, T. A role for PacMYBA in ABA-regulated anthocyanin biosynthesis in red-colored sweet cherry cv. Hong Deng (Prunus avium L.). Plant Cell Physiol. 2014, 55, 862-880. [CrossRef]

20. Kadomura-Ishikawa, Y.; Miyawaki, K.; Takahashi, A.; Masuda, T.; Noji, S. Light and abscisic acid independently regulated FaMYB10 in Fragaria $x$ ananassa fruit. Planta 2015, 241, 953-965. [CrossRef]

21. Fontana, A.R.; Antoniolli, A.; Bottini, R. Grape pomace as a sustainable source of bioactive compounds: Extraction, characterization, and biotechnological applications of phenolics. J. Agric. Food Chem. 2013, 61, 8987-9003. [CrossRef]

22. Wang, F.; Zhang, X.; Yang, Q.; Zhao, Q. Exogenous melatonin delays postharvest fruit senescence and maintains the quality of sweet cherries. Food Chem. 2019, 301, 125311. [CrossRef]

23. Meng, J.F.; Xu, T.F.; Song, C.Z.; Yu, Y.; Hu, F.; Zhang, L.; Zhang, Z.W.; Xi, Z.M. Melatonin treatment of pre-veraison grape berries to increase size and synchronicity of berries and modify wine aroma components. Food Chem. 2015, 185, 127-134. [CrossRef] [PubMed]

24. Hilbert, G.; Temsamani,H.; Bordenave, L.; Pedrot, E.; Chaher, N.; Cluzet, S.; Delaunay, J.C.; Ollat, N.; Delrot, S.; Merillon, J.M.; et al. Flavonol profiles in berries of wild Vitis accessions using liquid chromatography coupled to mass spectrometry and nuclear magnetic resonance spectrometry. Food Chem. 2015, 169, 49-58. [CrossRef] [PubMed]

25. Meng, J.F.; Yu, Y.; Shi, T.C.; Fu, Y.S.; Zhao, T.; Zhang, Z.W. Melatonin treatment of pre-veraison grape berries modifies phenolic components and antioxidant activity of grapes and wine. Food Sci. Technol. 2019, 39, 35-42. [CrossRef]

26. Debnath, B.; Hussain, M.; Li, M.; Lu, X.; Sun, Y.; Qiu, D. Exogenous melatonin improves fruit quality features, health promoting antioxidant compounds and yield traits in tomato fruits under acid rain stress. Molecules 2018, 23, 1868. [CrossRef]

27. Wu, Q.; Bai, J.; Tao, X.; Mou, W.; Luo, Z.; Mao, L.; Ban, Z.; Ying, T.; Li, L. Synergistic effect of abscisic acid and ethylene on color development in tomato (Solanum lycopersicum L.) fruit. Sci. Hortic. 2018, 235, 169-180. [CrossRef]

28. Olivares, D.; Contreras, C.; Munoz, V.; Rivera, S.; Gonzalez-Aguero, M.; Retamales, J.; Defilippi, B.G. Relationship among color development, anthocyanin and pigment-related gene expression in 'Crimson Seedless' grapes treated with abscisic acid and sucrose. Plant Physiol. Biochem. 2017, 115, 286-297. [CrossRef]

29. Zhang, Q.; Liu, Y.; He, C.; Zhu, S. Postharvest exogenous application of abscisic acid reduces internal browning in pineapple. J. Agric. Food Chem. 2015, 63, 5313-5320. [CrossRef]

30. Gao, Z.; Li, Q.; Li, J.; Chen, Y.; Luo, M.; Li, H.; Wang, J.; Wu, Y.; Duan, S.; Wang, L.; et al. Characterization of the ABA receptor VIPYL1 that regulates anthocyanin accumulation in grape berry skin. Front. Plant Sci. 2018, 9, 592. [CrossRef]

31. Shin, D.H.; Cho, M.; Choi, M.G.; Das, P.K.; Lee, S.K.; Choi, S.B.; Park, Y.I. Identification of genes that may regulate the expression of the transcription factor production of anthocyanin pigment 1 (PAP1)/MYB75 involved in Arabidopsis anthocyanin biosynthesis. Plant Cell Rep. 2015, 34, 805-815. [CrossRef] 
32. Wei, J.; Li, D.X.; Zhang, J.R.; Shan, C.; Rengel, Z.; Song, Z.B.; Chen, Q. Phytomelatonin receptor PMTR1-mediated signaling regulates stomatal closure in Arabidopsis thaliana. J. Pineal Res. 2018, 65, e12500. [CrossRef]

33. Xu, L.; Yue, Q.; Xiang, G.; Bian, F.; Yao, Y. Melatonin promotes ripening of grape berry via increasing the levels of ABA, $\mathrm{H}_{2} \mathrm{O}_{2}$, and particularly ethylene. Hortic. Res. 2018, 5, 41. [CrossRef] [PubMed]

34. Gao, H.; Lu, Z.; Yang, Y.; Wang, D.; Yang, T.; Cao, M.; Cao, W. Melatonin treatment reduces chilling injury in peach fruit through its regulation of membrane fatty acid contents and phenolic metabolism. Food Chem. 2018, 245, 659-666. [CrossRef] [PubMed]

35. Matus, J.T.; Poupin, M.J.; Canon, P.; Bordeu, E.; Alcalde, J.A.; Arce-Johnson, P. Isolation of WDR and bHLH genes related to flavonoid synthesis in grapevine (Vitis vinifera L.). Plant Mol. Biol. 2010, 72, 607-620. [CrossRef] [PubMed]

36. Gambino, G.; Perrone, I.; Gribaudo, I. A Rapid and effective method for RNA extraction from different tissues of grapevine and other woody plants. Phytochem. Anal. 2008, 19, 520-525. [CrossRef] [PubMed]

37. Deng, W.; Wang, Y.; Liu, Z.; Cheng, H.; Xue, Y. HemI: A toolkit for illustrating heatmaps. PLoS ONE 2014, 9, e111988. [CrossRef]

Sample Availability: Samples of the compounds are not available from the authors. 